and treatment with a neutralizing antibody against TNF prevented the tissue damage.

Two caveats should be noted. First, a developmental defect might make Timp $3^{-/-}$mice more prone to produce TNF, although the only aberration in development that has been observed is in the lung ${ }^{14}$. A conditional knockout will be required to rule out a developmental cause of the phenotype. Second, the evidence for elevated TACE activity, increased cleavage of a peptide substrate by liver homogenates from Timp $3^{-1-}$ mice, is not conclusive. Like all known peptide substrates of TACE, the one used by the authors can be cleaved by other proteases. The authors show convincingly that the increase in cleavage (i.e., beyond that observed with liver homogenates from wild-type mice) is blocked by an inhibitor of TACE (TIMP3, in fact) and is not due to other reported TNF-processing enzymes. Nonetheless, in the absence of specific TACE inhibitors and substrates, interpretation of these results requires caution.

The greater peptide cleavage by liver extracts from Timp $3^{-1-}$ mice also leads to an interesting question. If the increased cleavage is due to increased TACE activity, is this elevated activity due to relief from TIMP3 inhibition or to the presence of a greater amount of TACE protein? This question arises because the authors note that there is an increase in the amount of mature TACE (the enzyme itself requires processing for activation) in liver extracts of Timp $3^{-1-}$ mice. Another twist is that in the absence of TIMP3, there is not only more processed TNF but also more of the cell-associated form of the cytokine. One explanation for this is that, after an initial increase in TNF processing due to relief from TIMP3 inhibition of TACE, the resultant increase in soluble cytokine induces (directly or indirectly) the expression of TNF itself and the processing enzyme (Fig. 1). A more radical hypothesis is that TIMP3 normally downregulates TNF and TACE expression, possibly through a cell-surface receptor or by blocking release of an inducer (Fig. 1).

\section{Insights into inflammation}

Whatever the relevant target, how TIMP3 reaches it is not clear, because TIMP3 binds to extracellular matrix components ${ }^{1}$. It is also unclear why spontaneous inflammation in Timp $3^{-1-}$ mice occurs only in the liver and not in the lung, gut or skin-other sites where the mice may be exposed to inflammatory stimuli from the environment. Both of the TNF receptors (I and II) are also TACE substrates, and perhaps in some tissues the increased release of TNF is balanced by an increase in soluble receptors.

If we set aside the two caveats noted above, and accept the less radical explanation for the increase in mature TACE protein and total TNF production (Fig. 1), this work settles two lingering issues in the TNF field: is the generation of soluble TNF important, and is TACE the main physiological TNF-processing enzyme? The answer seems to be yes, in both cases.

More broadly, whatever the mechanism, this paper suggests that TIMP3 has a physiological role in regulating TNF and inflammation. This conclusion has implications for understanding and treating human diseases.
For example, as the authors note, chronic hepatitis in humans involves lymphocytic infiltration as seen in Timp $3^{-1-}$ mice. An examination of TIMP3 levels, TACE activity and TNF production in individuals with hepatitis could therefore lead to a better understanding of this disease, and these parameters should be considered in the study of other inflammatory conditions as well. Moreover, if a deficiency of TIMP3 is detrimental, could elevating its levels be beneficial in inflammatory conditions? Adenoviral delivery to rheumatoid synoviocytes has already been tested ${ }^{4}$, and at least one antiarthritic agent increases TIMP3 expres$\operatorname{sion}^{15}$. The work by Mohammed et al. ${ }^{5}$ should stimulate many more such investigations.

1. Staskus, P.W., Masiarz, F.R., Pallanck, L.J. \& Hawkes, S.P. J. Biol. Chem. 266, 449-454 (1991).

2. Baker, A.H., Zaltsman, A.B., George, S.J. \& Newby, A.C. J. Clin. Invest. 101, 1478-1487 (1998).

3. Qi, J.H. et al. Nat. Med. 9, 407-415 (2003).

4. Van der Laan, W.H. et al. Gene Therapy 10, 234-242 (2003).

5. Mohammed, F.F. et al. Nat. Genet. 36, 969-977 (2004)

6. Lambert, E., Dasse, E., Haye, B. \& Petitfrere, E. Crit. Rev. Oncol. Hematol. 49, 187-198 (2004).

7. Baker, A.H., Edwards, D.R. \& Murphy, G. J. Cell Sci. 115, 3719-3727 (2002).

8. Nagase, H. \& Woessner, J.F. Jr. J. Biol. Chem. 274 21491-21494 (1999).

9. Black, R.A. Int. J. Biochem. Cell Biol. 34, 1-5 (2002).

10. Moss, M.L. et al. Nature 385, 733-736 (1997).

11. Amour, A. et al. FEBS Lett. 435, 39-44 (1998).

12. Lee, M.-H. Biochem. J. 364, 227-234 (2002).

13. Wielockx, B. et al. Nat. Med. 7, 1202-1208 (2001).

14. Leco, K.J. et al. J. Clin. Invest. 108, 817-829 (2001).

15. Takizawa, M. et al. Arthritis Rheum. 43, 812-820 (2000).

\title{
The G-netics of dark skin
}

\author{
Ian J Jackson
}

Several mutant strains of mice have dark skin pigmentation due to an aberrant accumulation of pigment-producing melanocytes in the dermal layer of the skin. A new study shows that three such strains carry activating mutations in the genes encoding the Gprotein subunits $\mathrm{G} \alpha q$ or $\mathrm{G} \alpha 11$, resulting in more pigment cell precursors and an excess of dermally retained pigment cells at birth.

Mutations that affect mouse pigmentation have been studied for over a century. But despite this long history, large-scale mutagenesis programs ${ }^{1,2}$ continue to identify new genes involved in this process. A series of ten dominant dark skin (Dsk) mutations recently described by Greg Barsh and colleagues ${ }^{3}$ can

Ian J. Jackson is at the MRC Human Genetics Unit, Western General Hospital, Edinburgh, UK.e-mail:ian.jackson@hgu.mrc.ac.uk be classified into two categories, depending on whether dermal or epidermal pigmentation is increased. On page 961 of this issue, Van Raamsdonk et al. ${ }^{4}$ report that three of the four Dsk mutants with increased dermal pigmentation carry activating mutations in Gnaq and Gna11. These genes encode functionally related $\mathrm{G} \alpha$ subunits that mediate the first step in transducing signals from $G$ protein-coupled receptors, identifying a key role for these genes in regulating skin color.

\section{The road less traveled}

Melanoblasts, the precursors of the pigmentproducing cells of the skin and hair, arise during development from a migratory cell population called the neural crest. Some of the key molecules required in the developmental path from neural crest to melanoblast include the transcription factors Pax3, Sox10 and Mitf and the cell-surface receptors Kit and Ednrb ${ }^{5}$. As the two layers of the skin develop, melanoblasts enter the dermis and then 
migrate from the dermis to the epidermis through the basement membrane that separates them. Then, as hair follicles develop, melanoblasts undergo a further migration into the hair follicles, leaving the epidermis mostly devoid of pigment cells. In certain places where hair is sparse, such as the tail and external ears, melanocytes persist in the epidermis so that these structures become pigmented.

The developmental events regulating these migratory processes must be tightly controlled, but little is known about how this is achieved. One study suggested the cadherins have a role in this process: as dermal melanoblasts move through the basement membrane, they turn on E-cadherin, which is then downregulated and replaced by P-cadherin during migration into the hair follicles ${ }^{6}$. But our understanding of these regulatory events remains rudimentary.

\section{Mutant G proteins}

The new work from Greg Barsh and colleagues provides interesting insights into the regulation of melanoblast fate in the early embryo. As a starting point, Van Raamsdonk et al. adopted a positional cloning strategy to identify the genes underlying the Dsk1, Dsk7 and Dsk10 loci. They found that all three phenotypes were caused by mutations in genes encoding functionally related G-protein subunits: Dsk1 and Dsk10 are missense mutations in Gnaq, whereas Dsk7 is a missense mutation in Gna11.

These observations led to an obvious question: do these mutations produce a loss or gain of function in the resulting $G$ proteins? To address this point, the authors crossed the mutations onto Gnaq- or Gna11-null mutant backgrounds and showed that the Dsk phenotypes could be suppressed by loss of function of the other allele. A Dsk mutation in Gna11 could also be suppressed by loss of Gnaq function, and also vice versa, to some extent. These results indicate that the newly characterized $D s k$ mutations are hypermorphic, gain-of-function alleles that lead to an overstimulation of the signal transduction pathway. Furthermore, the dark skin phenotype showed a dose response to these hypermorphic alleles: as the number of Dsk alleles increased, the dermis became progressively darker (Fig. 1). In addition, the two $\mathrm{G} \alpha$ subunits seem to be interchangeable: the phenotype of a mouse with two hypermorphic Gna11 alleles was similar to that of a mouse with one hypermorphic Gna11 allele and one hypermorphic Gnaq allele.

Unexpectedly, the overactive G proteins encoded by the Dsk alleles do not seem to have a strong adverse effect on fitness. With

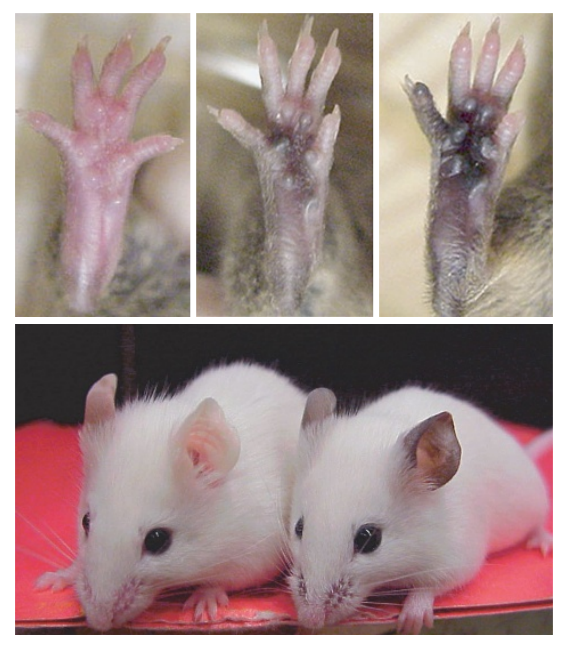

Photo courtesy of Greg Barsh

Figure 1 Photographs illustrating the dark skin phenotype of Dsk7 mice. The top panels show (from left to right) footpads of wild-type, Gna $11^{\text {Dsk7/+ }}$ and Gna $11^{\text {Dsk7/Desk7 mice. The }}$ dermal pigmentation increases with increasing copies of the mutated allele. The bottom panel shows mice homozygous with respect to the hypomorphic mutation $\mathrm{Kit}^{\mathrm{W}-\mathrm{v}}$. The mouse on the left is Gnal $1^{+/+}$, and the one on the right is Gna $11^{\text {Dsk7/+. }}$. The dark skin on the ears of the Dsk7 mouse shows that activated G-protein signaling can partially rescue the pigmentary defects caused by a reduction in Kit activity.

increasing mutant dose, mice became proportionately smaller, such that a mouse with four mutant copies is $\sim 25 \%$ underweight, but otherwise appears normal. This was somewhat unexpected, as transgenic mice with increased Gnaq expression in the heart have cardiac hypertrophy ${ }^{7}$.

What is the developmental basis for the increase in dermal pigmentation? Using a reporter gene ${ }^{8}$, the authors followed melanoblast development in Dsk mutant embryos and found an early excess of melanoblasts. But the number of melanoblasts that ultimately reached the epidermis and hair follicles was not affected by the mutations. It seems, then, that the migration of melanoblasts across the skin basement membrane is precisely regulated, such that only the correct number reaches the epidermis. In the mutants, however, the excess melanoblasts resulting from overstimulation of G-protein signaling are retained in the dermis, resulting in the dark skin phenotype.

\section{Receptor coupling}

Are these mutant $\mathrm{G} \alpha$ subunits constitutively active in isolation, or do they require coupling to receptor? The latter possibility seems more likely a priori, given the relatively mild phenotype of Dsk mice. Ednrb is a G protein-coupled receptor required for early melanoblast development ${ }^{9}$ and is known to couple to several $\mathrm{G} \alpha$ subunits, including $\mathrm{G} \alpha 11$ and $\mathrm{G} \alpha \mathrm{q}$ (ref. 10). Van Raamsdonk et al. show that the virtual absence of melanocytes in Ednrb mice was not rescued by the Dsk mutations. In contrast, the Dsk mutations did partially rescue the pigmentary defects resulting from loss of Pax3 or Kit (Fig. 1).

The time at which Dsk embryos have increased numbers of melanoblasts correlates well with the crucial period during which Ednrb is required for their development ${ }^{9}$. Therefore, the Dsk mutations probably exert their effects through coupling of G $\alpha 11$ and Goq to this receptor. But the mutations have not been shown to act autonomously in melanoblasts. It is possible that the $\mathrm{G} \alpha$ proteins are active in neighboring dermal cells, which then signal to the melanoblasts to affect their behavior. Indeed, two dark skin mutations that cause increased epidermal pigmentation are due to changes in genes encoding keratin and EGF-receptor, resulting in hyperkeratosis and thickening of the epidermis, and in these instances, hyperpigmentation is a secondary effect ${ }^{3}$. This caveat aside, melanoblasts from the mutant mice may be extremely valuable in dissecting the signaling pathways downstream of Ednrb. If the proliferative effects of the receptor are indeed

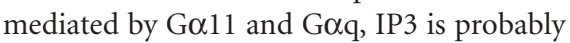
the intracellular messenger, leading to calcium release and activation of the MAPK/ERK pathway through $\mathrm{Src}^{10}$.

Despite decades of research on coat-color genes, mutagenic screens continue to identify new dominant mutations that affect pigmentation. Recessive screens will probably identify many more, particularly if we can identify those lethal mutations that affect the early stages of neural crest development. Few such mutations have been described, and there is a rich vein to be mined.

1. Nolan, P.M. et al. Nat. Genet. 25, 440-443 (2000).

2. Hrabe de Angelis, M.H. et al. Nat. Genet. 25, 444-447 (2000).

3. Fitch, K.R. et al. Genes Dev. 17, 214-228 (2003).

4. Van Raamsdonk, C.A. et al. Nat. Genet. 36, 961-968 (2004).

5. Barsh, G.S. Trends Genet. 12, 299-305 (1996).

6. Nishimura, E.K. et al. Dev. Biol. 215, 155-166 (1999).

7. D'Angelo, D.D. et al. Proc. Natl. Acad. Sci. USA 94, 8121-8126 (1997).

8. Mackenzie, M.A. et al. Dev. Biol. 192, 99-107 (1997).

9. Shin, M.K. et al. Nature 402, 496-501 (1999).

10. Cramer, H. et al. Eur. J. Biochem. 268, 5449-5459 (2001) 